\title{
Ramanujan's cubic continued fraction revisited
}

by

Heng Huat Chan and Kok Ping Loo (Singapore)

1. Introduction. Let $q=e^{2 \pi i \tau}$ and

$$
G(q)=\frac{q^{1 / 3}}{1}+\frac{q+q^{2}}{1}+\frac{q^{2}+q^{4}}{1}+\frac{q^{3}+q^{6}}{1} \ldots .
$$

In 1995, inspired by page 366 of Ramanujan's Lost Notebook [8], H. H. Chan [5] derived several new relations satisfied by $G(q)$. For example, he showed that

$$
G^{3}(q)=G\left(q^{3}\right) \frac{1-G\left(q^{3}\right)+G^{2}\left(q^{3}\right)}{1+2 G\left(q^{3}\right)+4 G^{2}\left(q^{3}\right)} .
$$

From (1.1), Chan constructed an algorithm for computing $e^{\pi}$. This iteration prompted F. G. Garvan to ask if there were any iteration to $\pi$ which can be derived from the study of $G(q)$. In this paper, we will show that such an iteration exists. We will also derive the following series for $1 / \pi$ :

$$
\frac{1}{\pi}=\frac{3 \sqrt{3}(3-2 \sqrt{2})}{2} \sum_{k=0}^{\infty} C_{k}\left(k+1-\frac{2}{3} \sqrt{2}\right)\left(-1+\frac{3}{4} \sqrt{2}\right)^{k}
$$

where

$$
C_{k}=\sum_{m=0}^{k}\left\{\sum_{j=0}^{m}\left(\begin{array}{c}
m \\
j
\end{array}\right)^{3} \sum_{i=0}^{k-m}\left(\begin{array}{c}
k-m \\
i
\end{array}\right)^{3}\right\} .
$$

The proof of (1.2) involves the identity

$$
G^{3}\left(e^{-2 \pi / \sqrt{6}}\right)=-1+\frac{3}{4} \sqrt{2} .
$$

Remarks. 1 . The function $G(q)$ can be expressed as

$$
\frac{\eta^{3}(6 \tau) \eta(\tau)}{\eta^{3}(3 \tau) \eta(2 \tau)}
$$

2000 Mathematics Subject Classification: 11Y65, 11F03. 
where $\eta(\tau)$ is defined by

$$
\eta(\tau)=q^{1 / 24} \prod_{k=1}^{\infty}\left(1-q^{k}\right), \quad q=e^{2 \pi i \tau} .
$$

However, we will not use this fact in this article.

2 . The series (1.2) converges slowly to $1 / \pi$. For every five terms in the series, we obtain roughly one additional correct decimal place for the decimal expansion of $1 / \pi$.

2. A triplication formula for $G(q)$ and a new iteration for $1 / \pi$. In [1], C. Adiga, T. Kim, M. S. M. Naika and H. S. Madhusudhan gave a new proof of (1.1) by first proving the identity

$$
1-3 \frac{G\left(q^{3}\right)}{1+G\left(q^{3}\right)}=\left(1-9 \frac{G^{3}(q)}{1+G^{3}(q)}\right)^{1 / 3} .
$$

This identity allows one to write $G\left(q^{3}\right)$ in terms of $G(q)$, namely,

$$
G\left(q^{3}\right)=\frac{1-H(q)}{2+H(q)}
$$

with

$$
H(q)=\left(\frac{1-8 G^{3}(q)}{1+G^{3}(q)}\right)^{1 / 3} .
$$

The above triplication formula for $G(q)$ is analogous to the Borweins-Ramanujan triplication formula for the cubic singular modulus defined by

$$
\frac{1}{\alpha(q)}=1+\frac{1}{27}\left(\frac{\eta(\tau)}{\eta(3 \tau)}\right)^{12}
$$

where $q=e^{2 \pi i \tau}$ and $\eta(\tau)$ is defined in (1.4). In the case of $\alpha(q)$, the triplication formula is given by

$$
\alpha\left(q^{3}\right)=\left(\frac{1-\sqrt[3]{1-\alpha(q)}}{1+2 \sqrt[3]{1-\alpha(q)}}\right)^{3} .
$$

Two rapidly convergent sequences for $\pi$ can be constructed from (2.4). These iterations are given as follows:

The Borweins Iteration [4]. Let $t_{0}=1 / 3, s_{0}=(\sqrt{3}-1) / 2$,

$$
s_{n}=\frac{1-\left(1-s_{n-1}^{3}\right)^{1 / 3}}{1+2\left(1-s_{n-1}^{3}\right)^{1 / 3}}, \quad t_{n}=\left(1+2 s_{n}\right)^{2} t_{n-1}-3^{n-1}\left(\left(1+2 s_{n}\right)^{2}-1\right) .
$$

Then $t_{n}^{-1}$ converges cubically to $\pi$. 
Chan's iteration [7]. Let $k_{0}=0, s_{0}=1 / 2^{1 / 3}$,

$$
s_{n}=\frac{1-\left(1-s_{n-1}^{3}\right)^{1 / 3}}{1+2\left(1-s_{n-1}^{3}\right)^{1 / 3}}, \quad k_{n}=\left(1+2 s_{n}\right)^{2} k_{n-1}+8 \cdot 3^{n-2} \sqrt{3} s_{n} \frac{1-s_{n}^{3}}{1+2 s_{n}} .
$$

Then $k_{n}^{-1}$ converges cubically to $\pi$.

Since the above iterations are constructed from (2.4), it is therefore natural to construct a new cubic iteration tending to $\pi$ from (2.2). In the following two sections, we will establish the following result:

Theorem 2.1. Let $k_{0}=0$ and $s_{0}=\sqrt[3]{\frac{3 \sqrt{2}}{4}-1}$. Set

$$
s_{n}=\frac{\left(1+s_{n-1}^{3}\right)^{1 / 3}-\left(1-8 s_{n-1}^{3}\right)^{1 / 3}}{2\left(1+s_{n-1}^{3}\right)^{1 / 3}+\left(1-8 s_{n-1}^{3}\right)^{1 / 3}} .
$$

If

$$
\begin{aligned}
k_{n}= & \frac{\left(1+2 s_{n}+4 s_{n}^{2}\right)\left(1+s_{n}\right)^{2}}{1-s_{n}+s_{n}^{2}} k_{n-1} \\
& +\frac{2 \cdot 3^{n-1}}{\sqrt{6}} \frac{s_{n}\left(1-2 s_{n}\right)\left(8 s_{n}^{4}-10 s_{n}^{3}+6 s_{n}^{2}+11 s_{n}+5\right)}{1+s_{n}^{3}},
\end{aligned}
$$

then $k_{n}^{-1}$ converges cubically to $\pi$.

REMARK. The values of $1 / k_{2}, 1 / k_{3}$ and $1 / k_{4}$ give $\pi$ correct to 7,27 and 86 decimal places, respectively.

3. New identities satisfied by $G(q)$. We first relate $G(q)$ with the Borweins' cubic singular modulus $\alpha(q)$ (see (2.3)) and deduce results on $G(q)$ using Ramanujan-Borweins' theory of elliptic functions to the cubic base [3].

Lemma 3.1. Let

$$
\begin{array}{ll}
\varphi(q)=\sum_{n=-\infty}^{\infty} q^{n^{2}}, & X=G^{3}(q), \\
a(q)=\sum_{m, n=-\infty}^{\infty} q^{m^{2}+m n+n^{2}}, & z=\frac{\varphi^{3}\left(-q^{3}\right)}{\varphi(-q)} .
\end{array}
$$

Then

$$
\begin{aligned}
a(q) & =z(1+4 X), \\
\alpha(q) & =27 \frac{X}{(1+4 X)^{3}} .
\end{aligned}
$$

Proof. From [2, p. 460, Entry 3(ii)], we find that

$$
a\left(q^{2}\right)=\frac{\varphi^{4}(-q)+3 \varphi^{4}\left(-q^{3}\right)}{4 \varphi(-q) \varphi\left(-q^{3}\right)}=z\left(\frac{1}{4} \frac{\varphi^{4}(-q)}{\varphi^{4}\left(-q^{3}\right)}+\frac{3}{4}\right) .
$$


Since [2, p. 347]

$$
\frac{\varphi^{4}(-q)}{\varphi^{4}\left(-q^{3}\right)}=1-8 X
$$

we deduce that

$$
a\left(q^{2}\right)=z(1-2 X) .
$$

On the other hand, we know that [3, p. 4189]

$$
a(q)=3 \frac{\varphi^{3}\left(-q^{3}\right)}{\varphi(-q)}-2 a\left(q^{2}\right) .
$$

Hence, by (3.4), we find that

$$
a(q)=z(1+4 X),
$$

which yields (3.1).

To prove (3.2), we recall the identity [2, p. 345, Entry 1(iv)]

$$
1+\frac{1}{27}\left(\frac{\eta(\tau)}{\eta(3 \tau)}\right)^{12}=\frac{(1+4 X)^{3}}{27 X}
$$

Using (2.3), we immediately deduce (3.2).

COROLlary 3.2. The functions $z$ and $X$ satisfy the following differential equations:

$$
q \frac{d X}{d q}=z^{2}\left(X-7 X^{2}-8 X^{3}\right) .
$$

Proof. We recall the differential equation satisfied by $a:=a(q)$ and $\alpha:=\alpha(q)[6,(4.7)]:$

$$
q \frac{d \alpha}{d q}=a^{2} \alpha(1-\alpha)
$$

Differentiating (3.2) with respect to $q$ and using (3.6) and (3.1), we immediately deduce (3.5).

4. Proof of Theorem 2.1. We begin our proof with the following transformation formula:

$$
\left(1+X\left(e^{-2 \pi / \sqrt{6 t}}\right)\right)\left(1+X\left(e^{-2 \pi \sqrt{t / 6}}\right)\right)=9 / 8
$$

This identity can be proved by rearranging the identity [1]

$$
\left(1+\frac{1}{X\left(e^{-2 \pi / \sqrt{6 t}}\right)}\right)\left(1-8 X\left(e^{-2 \pi \sqrt{t / 6}}\right)\right)=9 .
$$

Differentiating (4.1) with respect to $t$ and using (3.5), we find that

$$
\begin{aligned}
t Z\left(e^{-2 \pi \sqrt{t / 6}}\right) X\left(e^{-2 \pi \sqrt{t / 6}}\right)\left(1-8 X\left(e^{-2 \pi \sqrt{t / 6}}\right)\right) \\
=Z\left(e^{-2 \pi / \sqrt{6 t}}\right) X\left(e^{-2 \pi / \sqrt{6 t}}\right)\left(1-8 X\left(e^{-2 \pi / \sqrt{6 t}}\right)\right),
\end{aligned}
$$


where

$$
Z(q)=z^{2}
$$

From (4.2), we have

$$
\begin{aligned}
X\left(e^{-2 \pi / \sqrt{6 t}}\right) & =\frac{1}{9}\left(1+X\left(e^{-2 \pi / \sqrt{6 t}}\right)\right)\left(1-8 X\left(e^{-2 \pi \sqrt{t / 6}}\right)\right), \\
X\left(e^{-2 \pi \sqrt{t / 6}}\right) & =\frac{1}{9}\left(1+X\left(e^{-2 \pi \sqrt{t / 6}}\right)\right)\left(1-8 X\left(e^{-2 \pi / \sqrt{6 t}}\right)\right) .
\end{aligned}
$$

Substituting (4.4) and (4.5) into (4.3), we find that

$$
t Z\left(e^{-2 \pi \sqrt{t / 6}}\right)\left(1+X\left(e^{-2 \pi \sqrt{t / 6}}\right)\right)=Z\left(e^{-2 \pi / \sqrt{6 t}}\right)\left(1+X\left(e^{-2 \pi / \sqrt{6 t}}\right)\right) .
$$

This transformation formula motivates us to set

$$
A(q)=Z(q)(1+X(q))
$$

We can then express (4.6) as

$$
t A\left(e^{-2 \pi \sqrt{t / 6}}\right)=A\left(e^{-2 \pi / \sqrt{6 t}}\right) .
$$

Define

$$
\kappa(t)=\frac{1}{\pi A\left(e^{-2 \pi \sqrt{t / 6}}\right)}-2 \sqrt{\frac{t}{6}} \frac{\widetilde{A}}{A^{2}}\left(e^{-2 \pi \sqrt{t / 6}}\right),
$$

where

$$
\widetilde{f}:=q \frac{d f}{d q} .
$$

Differentiating both sides of (4.7) with respect to $t$, we find that

$$
\sqrt{\frac{t}{6}} \frac{\tilde{A}}{A}\left(e^{-2 \pi \sqrt{t / 6}}\right)+\sqrt{\frac{1}{6 t}} \frac{\tilde{A}}{A}\left(e^{-2 \pi / \sqrt{6 t}}\right)=\frac{1}{\pi} .
$$

Rewriting (4.9) in terms of $\kappa(t)$ yields

$$
\kappa(t)+t \kappa(1 / t)=0 .
$$

When $t=1$, (4.10) implies that

$$
\kappa(1)=0
$$

Next, let

$$
M_{N}(q)=A(q) / A\left(q^{N}\right)
$$

Setting $q=e^{-2 \pi \sqrt{t / 6}}$ and differentiating (4.12) with respect to $t$, we find using (4.8) that

$$
\begin{aligned}
& \kappa\left(N^{2} t\right) \\
& \quad=2 \sqrt{\frac{t}{6}} \frac{\widetilde{M}_{N}}{M_{N}}\left(e^{-2 \pi \sqrt{t / 6}}\right) \frac{1}{A\left(e^{-2 \pi \sqrt{N^{2} t / 6}}\right)}-M_{N}\left(e^{-2 \pi \sqrt{t / 6}}\right) \kappa(t) .
\end{aligned}
$$

Note that $\kappa\left(N^{2 l} t\right)$ tends to $1 / \pi$ at the rate of order $N$ as $l$ tends to $\infty$. 
In order to obtain a cubic iteration tending to $1 / \pi$ from (4.13), let $N=3$. If $y=G\left(q^{3}\right)$ then from $[5,(2.9)]$, we have

$$
\frac{\varphi\left(-q^{9}\right)}{\varphi(-q)}=\frac{1}{1-2 y}
$$

Using (3.3) and (4.14), we deduce that

$$
\begin{aligned}
\frac{Z(q)}{Z\left(q^{3}\right)} & =\frac{\varphi^{6}\left(-q^{3}\right)}{\varphi^{2}(-q)} \frac{\varphi^{2}\left(-q^{3}\right)}{\varphi^{6}\left(-q^{9}\right)}=\frac{\varphi^{8}\left(-q^{3}\right)}{\varphi^{8}\left(-q^{9}\right)} \frac{\varphi^{2}\left(-q^{9}\right)}{\varphi^{2}(-q)} \\
& =\left(\frac{1-8 y^{3}}{1-2 y}\right)^{2}=\left(1+2 y+4 y^{2}\right)^{2} .
\end{aligned}
$$

Hence,

$$
M_{3}=\left(1+2 y+4 y^{2}\right)^{2} \frac{1+X}{1+y^{3}}=\frac{\left(1+2 y+4 y^{2}\right)(1+y)^{2}}{1-y+y^{2}},
$$

by (1.1).

Using (3.5) with $q$ replaced by $q^{3}$, we have

$$
\widetilde{y}=A\left(q^{3}\right) y\left(1-8 y^{3}\right) \text {. }
$$

This allows us to differentiate both sides of (4.15) and conclude that

$$
\frac{1}{M_{3}(q) A\left(q^{3}\right)} \widetilde{M}_{3}(q)=\frac{(1-2 y) y\left(8 y^{4}-10 y^{3}+6 y^{2}+11 y+5\right)}{(y+1)\left(1-y+y^{2}\right)} .
$$

We are now ready to construct our sequence $k_{n}$. Let $s_{n}=G\left(e^{-2 \pi \sqrt{3^{2 n} / 6}}\right)$ and $k_{n}=\kappa\left(3^{2 n}\right)$. Writing (4.13) in terms of $s_{n}$ and $k_{n}$, we find that

$$
\begin{aligned}
k_{n}= & \frac{\left(1+2 s_{n}+4 s_{n}^{2}\right)\left(1+s_{n}\right)^{2}}{1-s_{n}+s_{n}^{2}} k_{n-1} \\
& +\frac{2 \cdot 3^{n-1}}{\sqrt{6}} \frac{s_{n}\left(1-2 s_{n}\right)\left(8 s_{n}^{4}-10 s_{n}^{3}+6 s_{n}^{2}+11 s_{n}+5\right)}{1+s_{n}^{3}} .
\end{aligned}
$$

From (4.11), we know that the initial value of $k_{n}$ is $k_{0}=0$. By letting $t=1$ in (4.1), we find that the initial value of $s_{0}$ is

$$
s_{0}=G\left(e^{-2 \pi / \sqrt{6}}\right)=(3 \sqrt{2} / 4-1)^{1 / 3} .
$$

We can then evaluate $s_{n}$ from $s_{n-1}$ using (2.2). Substituting $s_{n}$ into (4.17), we construct the sequence $\left\{k_{n}\right\}$ which converges cubically to $1 / \pi$ and this completes the proof of Theorem 2.1.

5. A series for $1 / \pi$. Set $t=1$ in (4.9). We find that

$$
\frac{\widetilde{A}}{A}\left(e^{-2 \pi / \sqrt{6}}\right)=\frac{\sqrt{6}}{2 \pi}
$$


Using the relations (3.1) and (3.2) in the differential equation $\left(^{1}\right)$

$$
\alpha(1-\alpha) \frac{d^{2} a}{d \alpha^{2}}+(1-2 \alpha) \frac{d a}{d \alpha}-\frac{2}{9} a=0
$$

we deduce that

$$
X(8 X-1)(1+X) \frac{d^{2} z}{d X^{2}}+\left(24 X^{2}+14 X-1\right) \frac{d z}{d X}+2(1+4 X) z=0
$$

If

$$
z=\sum_{k=0}^{\infty} c_{k} X^{k}
$$

then from (5.2), we know that $a_{k}$ satisfies the recurrence

$$
k^{2} c_{k}-\left(7 k^{2}-7 k+2\right) c_{k-1}-8(k-1)^{2} c_{k-2}=0 .
$$

The solution of the above recurrence with $c_{0}=1, c_{1}=2$ is given by $[9$, Table 2] $\left({ }^{2}\right)$

$$
c_{k}=\sum_{j=0}^{k}\left(\begin{array}{l}
k \\
j
\end{array}\right)^{3}
$$

Hence,

$$
z=\sum_{k=0}^{\infty} \sum_{j=0}^{k}\left(\begin{array}{l}
k \\
j
\end{array}\right)^{3} X^{k}
$$

Therefore,

$$
Z=z^{2}=\sum_{k=0}^{\infty} C_{k} X^{k}
$$

where $C_{k}$ is given by (1.3), or

$$
A=\sum_{k=0}^{\infty} C_{k} X^{k}(1+X)
$$

From (5.3), we deduce that

$$
\frac{\widetilde{A}}{A}=\frac{1}{A} \frac{d A}{d X} \widetilde{X}=(1-8 X) \sum_{k=0}^{\infty} C_{k} X^{k}(k(1+X)+X),
$$

by $(3.5)$.

Set $q=e^{-2 \pi / \sqrt{6}}$ in (5.4). From (4.18), we know that

$$
X\left(e^{-2 \pi / \sqrt{6}}\right)=x_{1}=-1+3 \sqrt{2} / 4 .
$$

$\left({ }^{1}\right)$ See [6] for a derivation of this differential equation and its solutions.

$\left({ }^{2}\right)$ According to H. A. Verrill, the solution to the recurrence is due to D. Zagier. 
Hence, we have

$$
\left(1-8 x_{1}\right) \sum_{k=0}^{\infty} C_{k} x_{1}^{k}\left(k\left(1+x_{1}\right)+x_{1}\right)=\frac{\sqrt{6}}{2 \pi} .
$$

Simplifying the above yields (1.2).

6. Concluding remarks. 1 . We have seen here that (4.1) plays an important role for our determination of $A(q)$. In general, if we have a modular function (i.e. a Hauptmodul) associated to a congruence subgroup $\Gamma$ of $S L_{2}(\mathbb{Z})$ with genus zero, we need to determine a "nice" modular form of weight 2 on $\Gamma$ in order to derive new series for $1 / \pi$. It is therefore possible to derive new series for $1 / \pi$ associated with the Rogers-Ramanujan continued fraction.

2. We can also obtain another cubic iteration tending to $1 / \pi$ if we use the alternative formula [1]

$$
\left(1+\frac{1}{G^{3}\left(-e^{-\pi t}\right)}\right)\left(1+\frac{1}{G^{3}\left(-e^{-\pi / t}\right)}\right)=9 .
$$

We leave this as an exercise for the readers.

Acknowledgements. The first author thanks sincerely S. Cooper and the Institute for Information and Mathematical Sciences, Massey University (Albany Campus) for their generous support during his stay there. We are also grateful to W. C. Liaw and the referee for pointing out some misprints in an earlier version of this article.

\section{References}

[1] C. Adiga, T. Kim, M. S. M. Naika and H. S. Madhusudhan, On Ramanujan's cubic continued fraction and explicit evaluations of theta-functions, Indian J. Pure Appl. Math. 35 (2004), 1047-1062.

[2] B. C. Berndt, Ramanujan's Notebooks, Part III, Springer, New York, 1991.

[3] B. C. Berndt, S. Bhargava and F. G. Garvan, Ramanujan's theories of elliptic functions to alternative bases, Trans. Amer. Math. Soc. 347 (1995), no. 11, 4163-4244.

[4] J. M. Borwein and F. G. Garvan, Approximations to $\pi$ via the Dedekind eta functions, CMS Conf. Proc. on Organic Math. (Burnaby, BC), 20, Amer. Math. Soc., Providence, RI, 1987, 89-115.

[5] H. H. Chan, On Ramanujan's cubic continued fraction, Acta Arith. 73 (1995), 343355 .

[6] -, On Ramanujan's cubic transformation formula for ${ }_{2} F_{1}\left(\frac{1}{3}, \frac{2}{3} ; 1 ; z\right)$, Math. Proc. Cambridge Philos. Soc. 124 (1998), 193-204.

[7] - Ramanujan's elliptic functions to alternative bases and approximations to $\pi$, in: Number Theory for the Millennium I (Urbana, IL, 2000), A K Peters, 2002, 197-213. 
[8] S. Ramanujan, The Lost Notebook and Other Unpublished Papers, Narosa, New Delhi, 1988.

[9] H. A. Verrill, Some congruences related to modular forms, preprint.

Department of Mathematics

National University of Singapore

2 Science Drive 2, Singapore 117543

E-mail: matchh@nus.edu.sg

Received on 11.7.2005

and in revised form on 29.11.2006 